\title{
Optical Gain from the Open Form of a Photochromic Molecule in the Solid State
}

\author{
Dario Pisignano, ${ }^{*},+, *$ Elisa Mele, ${ }^{\dagger, *}$ Luana Persano, ${ }^{\dagger, *}$ Athanassia Athanassiou, ${ }^{\dagger}$ \\ Costas Fotakis, ${ }^{\dagger}$ and Roberto Cingolani ${ }^{*}$ \\ Foundation for Research and Technology-Hellas (FORTH), Institute of Electronic Structure and Laser (IESL), \\ 711 10, Heraklion, Crete, Greece, and National Nanotechnology Laboratory of INFM-CNR and Agilent \\ Technologies Joint Lab, Department of Engineering of Innovation, University of Lecce, via Arnesano, \\ I-73100 Lecce, Italy
}

Received: January 9, 2006; In Final Form: January 27, 2006

\begin{abstract}
This work presents evidence for line-narrowing from the UV photoexcited open form of the photochromic molecule, indolinospiropyran $\left(1^{\prime}, 3^{\prime}\right.$-dihydro-1', $3^{\prime}, 3^{\prime}$-trimethyl-6-nitrospiro [2H-1-benzopyran-2,2'-(2H)-indole]) in the solid state. The line-narrowing is attributable to amplified spontaneous emission induced by optical gain and assisted by the waveguiding within the organic film. Optical gain throughout a band as large as 28 $\mathrm{nm}$, with a maximum gain coefficient of $5.6 \mathrm{~cm}^{-1}$, is observed in the merocyanine emission region (660$730 \mathrm{~nm}$ ). These results open the way to the realization of hybrid devices based on the coupling between photochromic behavior and stimulated emission from conjugated molecules, such as lasing optical memories, and lasers gated by optical molecular switches.
\end{abstract}

In the past decade, photochromic molecules ${ }^{1}$ have raised increasing interest by virtue of their possible application to optical switches ${ }^{2}$ and memories, ${ }^{3}$ three-dimensional data storage, ${ }^{4}$ and microfabrication. ${ }^{5}$ Photochromism, namely the reversible color change upon irradiation of suitable wavelength, is indeed often coupled with other light-controllable transformations of the molecular properties (particularly the electronic conjugation) due to the photoswitching between different states. Such features may include switchable absorption (suitable for memories), ${ }^{2,3}$ refractive index (for holography recording), ${ }^{6}$ wettability of surfaces (for microfluidic devices), ${ }^{7}$ and volume changes (for photoactuators). ${ }^{8}$ We propose that the photochromic behavior of some molecular systems can be also coupled with optical gain determined by the stimulated emission from one particular state.

In particular, spiropyran molecules, ${ }^{9}$ such as indolinospiropyran $\left(1^{\prime}, 3^{\prime}\right.$-dihydro-1', 3', 3'-trimethyl-6-nitrospiro [2H-1-benzopyran-2,2'-(2H)-indole], 6-nitro-BIPS) are initially nonpolar and transparent at visible wavelengths, and absorb in the ultraviolet (UV, $\lambda \leq 400 \mathrm{~nm}$ ), whereas they are reversibly converted into the isomeric form, trans-merocyanine, upon UV irradiation through the photochemical cleavage of the $\mathrm{C}-\mathrm{O}$ bond in the spiropyran ring (Figure 1). The colorless form can be recovered either by irradiation with green light, or thermally. The absorption spectrum of the colored form extends through visible wavelengths $(\lambda \leq 680 \mathrm{~nm})$. The merocyanine form indeed exhibits an extended $\pi$-electron delocalization with respect to the nonplanar BIPS system. The exploitation of such a $\pi$-conjugation of photochromic compounds for achieving stimulated emission would enable the design and implementation of a novel class of switching and memory devices, in which

\footnotetext{
* Corresponding author. E-mail: dario.pisignano@unile.it.

Institute of Electronic Structure and Laser (IESL).

$\doteqdot$ INFM-CNR at University of Lecce.
}

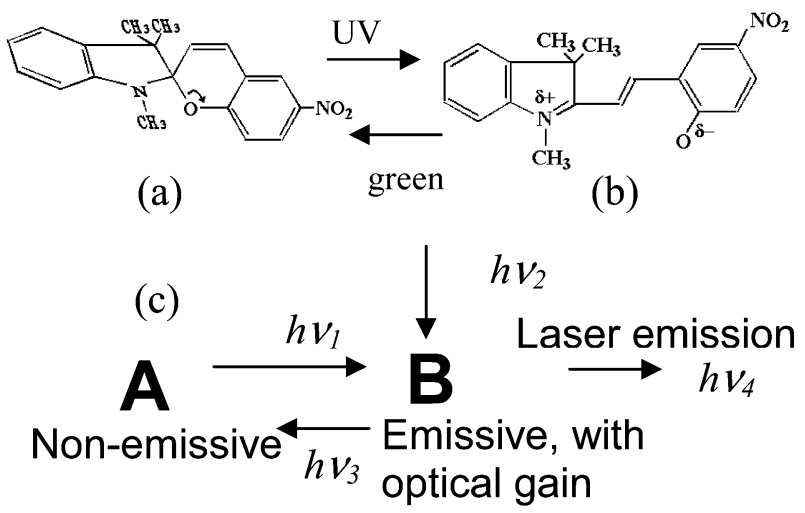

Figure 1. Reversible transformations between the closed (a) and the open (b) forms of the photochromic molecule (6-nitro-BIPS and merocyanine, respectively), by irradiation with UV and visible light. (c) Optical cycles for lasing optical memories or optically gated lasers.

one molecular state shows lasing behavior with high spectral purity and directional emission, and optically gateable solidstate lasers. Indeed, the interplay between photochromisms and the optical gain from one of the two molecular states allows one to envisage the optical cycle depicted in Figure 1c, in which one can achieve laser emission $\left(h v_{4}\right)$ by an excitation radiation $\left(h v_{2}\right)$ and activating and deactivating the lasing medium by $h v_{1}$ and $h v_{3}$, respectively.

In this work, we report on the observation of line-narrowing, ascribed to amplified spontaneous emission (ASE) and optical gain by the open form of a photochromic molecule, namely, the merocyanine resulting from the UV-irradiation of 6-nitroBIPS, embedded in a polymeric matrix at the solid state.

The photoluminescence (PL) spectra of 6-nitro-BIPS doped in poly(ethyl methacrylate)-co-poly(methyl acrylate) (PEMMA), under pumping by $3 \mathrm{~ns}$ pulses at $355 \mathrm{~nm}$, are displayed as a 


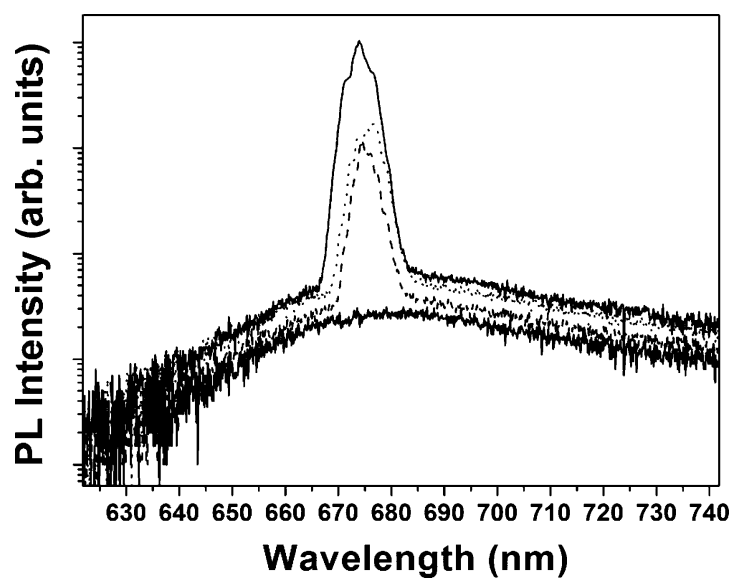

Figure 2. Emission spectra from the photochromic SP/merocyanines/ PEMMA films under different excitation fluences (from bottom to top: $0.3,2.5,3.7$, and $6.1 \mathrm{~mJ} \mathrm{~cm}^{-2}$ ). Sample thickness $\cong 695 \mathrm{~nm}$.

function of the excitation density in Figure 2. A featureless and broad (full width half-maximum, fwhm $\cong 60 \mathrm{~nm}$ ) spectrum is observable for low excitation fluences, $E$, whereas, for $E$ larger than about $400 \mu \mathrm{J} \mathrm{cm} \mathrm{cm}^{-2}$, a narrow peak at about $675 \mathrm{~nm}$ becomes more and more evident, resulting in a significant linenarrowing of the overall spectrum. Differently from previous experiments, ${ }^{8 \mathrm{c}}$ we could observe a quite narrow peak, exhibiting a width down to $3.3 \mathrm{~nm}$ (fwhm). This effect can be ascribed to ASE assisted by the waveguiding effect due to the different refractive index of substrate, gain medium, and vacuum. The output intensity exhibits a rapid (approximately exponential) ${ }^{10}$ increase up to about $4 \mathrm{~mJ} \mathrm{~cm}{ }^{-2}$, then it saturates for very large excitation intensities $\left(E>5 \mathrm{~mJ} \mathrm{~cm}^{-2}\right)$ because of the occurrence of nonradiative decay processes, whose contribution increases upon increasing the pump density. In our experiments, we also observed a remarkable dependence of the fluorescence spectral peak on the film thickness, with red-shifted line-narrowing in thicker samples, which is a signature of ASE behavior in conjugated organic materials. ${ }^{11}$ For instance, ASE was peaked at about 675 and $710 \mathrm{~nm}$ for films with thicknesses of 690 and $730 \mathrm{~nm}$, respectively.

The stimulated emission condition for conjugated compounds $^{12}$ is often described by a four-level scheme based on the vibronic progressions of the $S_{0}$ and $S_{1}$ states. The population inversion is indeed accomplished by the photoexcitation from the ground vibrational level of $S_{0}$ to a high-energy sublevel of $S_{1}$ and the subsequent nonradiative decay to the ground sublevel of $S_{1}$ by internal conversion. ${ }^{13}$ Basic prerequisites for such a process are a high luminescence efficiency ${ }^{14}$ and a large separation between the spectral regions of gain and absorption (native and photoinduced). Large Stokes' shift, hence low selfabsorption, strongly favors the lasing action. ${ }^{11}$ Such spectral separation for merocyanines can be as large as $0.5 \mathrm{eV}$.

Notwithstanding the importance of photochromic molecules for a wide number of potential applications, the mechanism of the BIPS-merocyanine photochemical conversion has not yet been fully assessed. A number of studies have found evidence of a transient intermediate (cis-merocyanine), leading to the rearrangement to the trans form in a short time $(<100 \mathrm{ps}){ }^{15}$ However, the electronic character (singlet or triplet) of the conversion and its temporal dynamics are not yet completely understood, although some theoretical ${ }^{16}$ and ultrafast (fs) ${ }^{17}$ studies have recently shed light on some issues. In our system, the UV excitation first determines an equilibrium condition between the native BIPS molecules and the open form, both present in the photoexcited sample. The spectral location of the

\section{SCHEME 1: Scheme of the Potential Energy Curve of the 6-Nitro-BIPS/Merocyanine System vs a Reaction Coordinate}

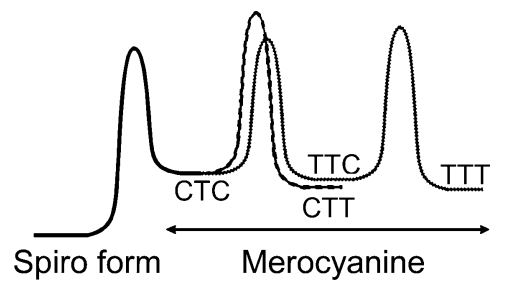

gain peak undoubtedly makes it assignable to merocyanine, possibly through the excitation to vibrationally hot states within $S_{1}$ or higher excited states $\left(S_{n}\right)$, or even the occurrence of different merocyanine isomer states (cis-trans or trans-trans), as proposed for 6,8-dinitro-BIPS in acetonitrile solutions,,${ }^{17 a}$ and ultimately the decay to the $S_{0}$ state of the open form.

A photocoloration yield of $\sim 0.6-0.8$ is reported for 6-nitroBIPS in solvents of low polarity. ${ }^{18}$ This photocoloration yield should correspond to the upper limit of the yield in our system, where the BIPS molecules are doped in PEMMA, which is a nonpolar but highly viscous medium. After the irradiation of 6-nitro-BIPS by UV light, the colorless form can be recovered either by irradiation with light of suitable wavelength, or thermally. However, the thermal recovery can be neglected for the time scales of our optical experiment. Indeed, Levitus et al. mention hundreds of minutes for thermal recovery of merocyanines to the spiropyran form in polymer matrixes at room temperature. ${ }^{19}$ In our experiments, we saw that the merocyanine samples were stable for many hours at room temperature.

Instead, the recovery induced by the continuous irradiation with pulsed UV light has to be taken into account, determining a nonzero back conversion rate, that is, a steady-state equilibrium (indicated by the stable PL signal that we obtained, given by the merocyanine form only) of the system under pumping conditions at $355 \mathrm{~nm}$. By the profile of the potential energy, associated with the conformational changes among the spiro form and the different merocyanine isomers ${ }^{20}$ and shown in Scheme 1, it is evident that irradiation with UV light can enable both coloration and reversion to the colorless form. In particular, the ring closure kinetics of some photochromic species, such as the 6,8-dinitro-BIPS and its carboxyethyl derivative, studied by pump-probe spectroscopy and time-resolved PL, could be explained in terms of a purely singlet-state manifold. ${ }^{17 a}$

To assess in depth the gain performances of the merocyanine, we carried out measurements by the variable stripe length method, which provides the optical gain spectrum, $g(\lambda)$, of the gain medium through the relation between the emitted intensity, $I$, and the excitation length, $l:{ }^{21} I(\lambda)=I_{0}(\lambda)\{\exp [g(\lambda) l]-1\}$. The measured gain spectrum, on a sample with a thickness of $\cong 730 \mathrm{~nm}$, shows a well-defined peak, centered at about 710 $\mathrm{nm}$ with a spectral width of $28 \mathrm{~nm}$ and a maximum of about $5.6 \mathrm{~cm}^{-1}$ (Figure 3). Such a value is comparable to that obtained in several conjugated oligomers and dye molecules, ${ }^{22}$ although it is still one order of magnitude lower than the state-of-the-art values achievable in poly(fluorene), poly(phenylenevinylene), and poly(paraphenylene) derivatives. ${ }^{23}$ Further experiments are currently in progress to achieve a complete description of the optical gain behavior and performances of merocyanines.

Overall, in the present work, the employed radiation $(\lambda=$ $355 \mathrm{~nm}$ ), induces both the conversion (and the recovery), and the excitation of the merocyanine states undergoing stimulated emission, namely, in our case, $h v_{1}=h v_{2}=h v_{3}$, according to Figure 1c. However, as indicated by Scheme 1, it is possible to 


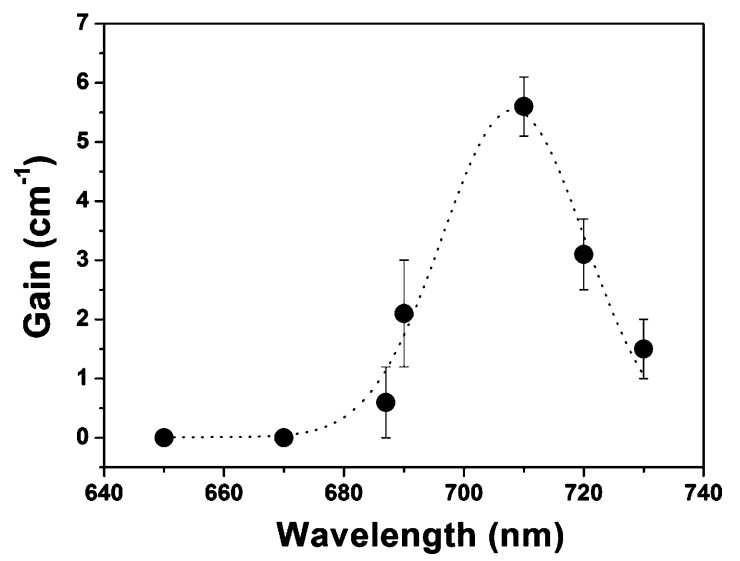

Figure 3. Optical gain spectrum for an excitation density of $0.5 \mathrm{~mJ}$ $\mathrm{cm}^{-2}$. The dotted line is a guide for the eye.

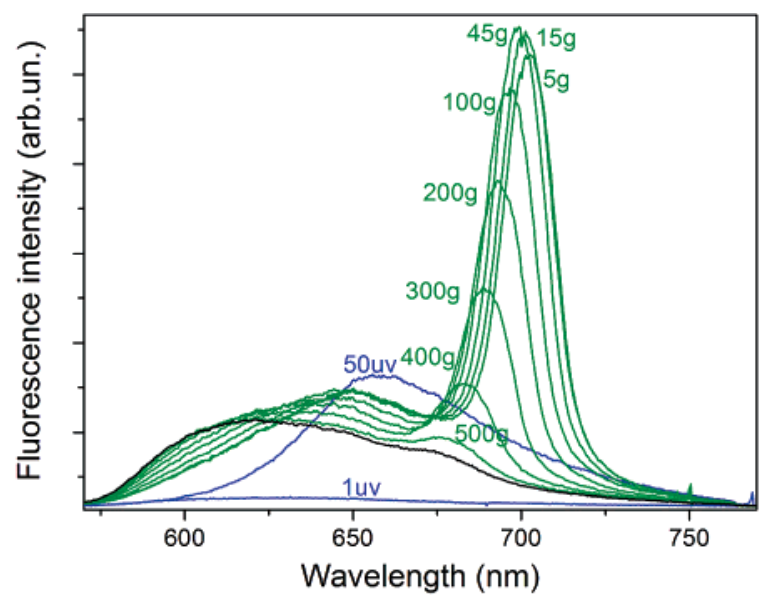

Figure 4. PL spectra of 6-nitro-BIPS/merocyanine/PEMMA samples, showing the activation of the merocyanine states (blue lines) by 50 initial UV pulses, and the subsequent recovery of the colorless spiro forms by $5,15,45,100,200,300,400$, and $500 \mathrm{~ns}$ green pulses (green lines, UV light fluence $=$ green light fluence $=45 \mathrm{~mJ} \mathrm{~cm}{ }^{-2}$ ). The reduction of both the line-narrowed peak and the spontaneous merocyanine fluorescence is observable upon green irradiation.

employ different wavelengths for the photocoloration and the back conversion from merocyanines and 6-nitro-BIPS, namely, UV and green light, respectively $\left(h v_{1} \neq h v_{3}\right)$. Figure 4 shows the fluorescence collected from 6-nitro-BIPS/merocyanines/ PEMMA samples, showing the activation of the merocyanine states by UV irradiation and the subsequent ring closure by green light, clearly indicating that the emission signal (and the optical gain of the system) can be switched off by optical control. This allows one to go beyond the conventional fields of nonphotochromic $\pi$-conjugated systems and photochromic compounds exhibiting spontaneous emission, and to gate the gain emission by optical control, which is not possible by nonphotochromic fluorescent $\pi$-conjugated systems.

Finally, we point out that several cycles can be performed by alternating exposure with UV and green light. The number of 6-nitro-BIPS/merocyanine cycles that can be performed depends on the thickness of the film, the percentage of the photochromic molecules in the sample, the fluence, and the number of pulses used to induce the conversion. For instance, by employing a UV and a green light fluence of 40 and $50 \mathrm{~mJ}$ $\mathrm{cm}^{-2}$, respectively, we could carry out 7 cycles of $50 \mathrm{UV}$ and 200 green pulses each, preserving active molecules within the sample (Figure 5). Although the absorbance contrast shows a reduction leading to a dampening of the cycles (Figure 5), we point out that the fluorescence signal from the merocyanine
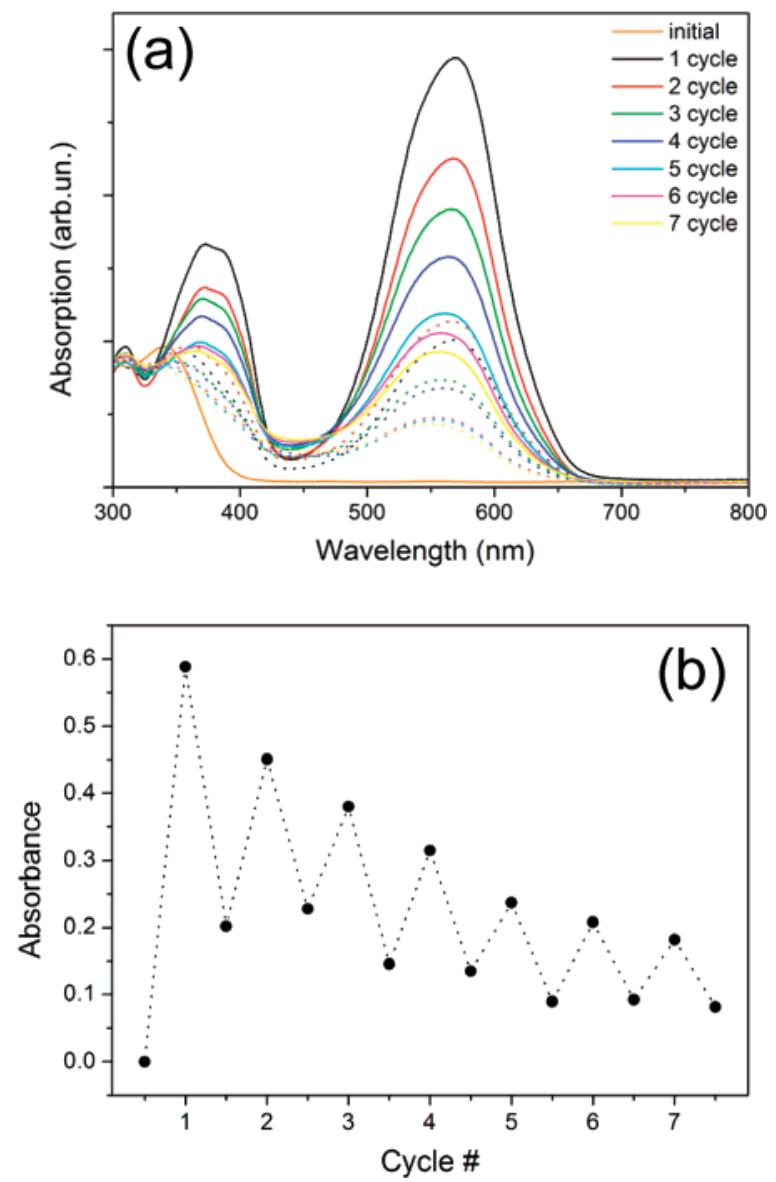

Figure 5. 6-Nitro-BIPS/merocyanine cycles performed by alternating $\mathrm{UV}$ and green irradiation (UV light fluence $=40 \mathrm{~mJ} \mathrm{~cm}^{-2}$; green light fluence $=50 \mathrm{~mJ} \mathrm{~cm}^{-2}$ ). (a) Absorption spectra of the system. The solid lines show the absorption spectra after UV irradiation, whereas the dotted lines show the spectra after green irradiation, in which the molecules recover back to the spiro form. (b) Absorbance at $568 \mathrm{~nm}$, vs the number of the performed cycle.

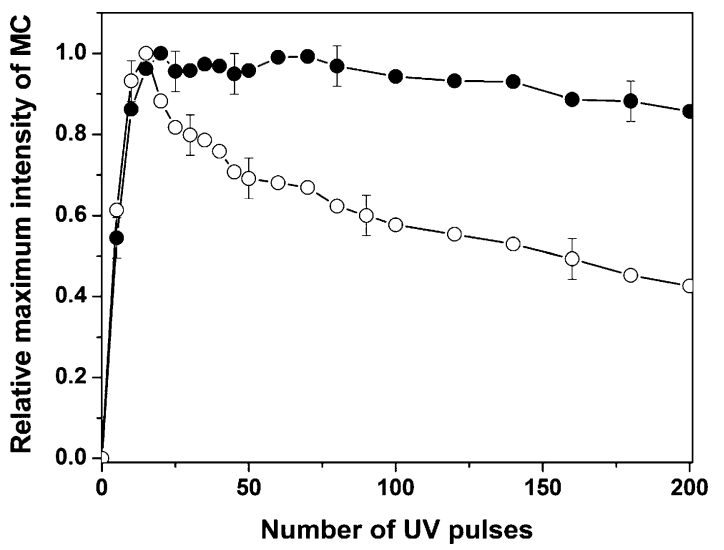

Figure 6. Observed reduction of the merocyanine (MC) absorption signal vs employed UV laser pulses, for excitation fluences of $8 \mathrm{~mJ}$ $\mathrm{cm}^{-2}$ (filled circles) and $15 \mathrm{~mJ} \mathrm{~cm}{ }^{-2}$ (open circles), respectively. Both fluences are well above the ASE threshold.

states can still be clearly recorded. In addition, the observed degradation of the merocyanine fluorescence can be significantly reduced by employing lower UV fluences, that are large enough to induce the stimulated emission in the system, by virtue of the low threshold fluence observed for ASE $\left(400 \mu \mathrm{J} \mathrm{cm}^{-2}\right)$. For instance, the degradation effect reduces the absorption of the merocyanine to $\sim 14 \%$ of the maximum value after $200 \mathrm{UV}$ laser pulses at $248 \mathrm{~nm}$ with a fluence of $8 \mathrm{~mJ} \mathrm{~cm}^{-2}$, whereas 
the reduction is $\sim 57 \%$ after 200 pulses with $15 \mathrm{~mJ} \mathrm{~cm}^{-2}$ (Figure $6)$. This demonstrates that the use of low laser fluences dramatically reduces the degradation effect. Several strategies can be envisaged, including the addition of oxygen-trapping molecules in the matrix, to further reduce the oxidative degradation.

In conclusion, we reported on line-narrowing, ascribable to ASE and optical gain from the photochromic compound, 6-nitroBIPS, in its open merocyanine form. The stimulated emission, evidenced by line-narrowing under pulsed UV excitation, is observable by the molecules at the solid state, with a maximum gain of $5.6 \mathrm{~cm}^{-1}$. This result opens the way to coupling photochromism and stimulated emission, that is, exploiting the lasing behavior of the open isomers to enhance the optical output of molecular memories, or, vice versa, gating laser devices by optical molecular switches.

Acknowledgment. D.P. acknowledges support from Marie Curie Research Training Networks, MRTN-CT-2003-505138 (EU 6th Framework Program).

Supporting Information Available: Full description of the experimental details. This material is available free of charge via the Internet at http://pubs.acs.org.

\section{References and Notes}

(1) (a) Yokoyama, Y. Chem. Rev. 2000, 100, 1717-1739. (b) Hampp, N. Chem. Rev. 2000, 100, 1755-1776. (c) Irie, M. Chem. Rev. 2000, 100, 1685-1716. (d) Tamai, N.; Miyasaka, H. Chem. Rev. 2000, 100, 18751890.

(2) (a) Willner, I. Acc. Chem. Res. 1997, 30, 347-356. (b) Cinelli, R. A. G.; Pellegrini, V.; Ferrari, A.; Faraci, P.; Nifosi, R.; Tyagi, M.; Giacca, M.; Beltram, F. Appl. Phys. Lett. 2001, 79, 3353-3355. (c) Raymo, F. N.; Giordani, S. Proc. Natl. Acad. Sci. U.S.A. 2002, 99, 4941-4944.

(3) (a) Tsivgoulis, G. M.; Lehn, J. M. Angew. Chem., Int. Ed. Engl. 1995, 34, 1119-1122. (b) Tyson, D. S.; Bignozzi, C. A.; Castellano F. N. J. Am. Chem. Soc. 2002, 124, 4562-4563. (c) Tsujioka, T.; Kondo, H. Appl. Phys. Lett. 2003, 83, 937-939. 845.

(4) Parthenopoulos, D. A.; Rentzepis, P. M. Science 1989, 245, 843-

(5) Ebisawa, F.; Hoshino, M.; Sukegawa, K. Appl. Phys. Lett. 1994, $65,2919-2920$.

(6) (a) Weiss., V.; Friesem, A. A.; Krongauz, V. A. Opt. Lett. 1993 18, 1089-1091. (b) Akella, A.; Sochava, S. L.; Hesselink L. Opt. Lett. 1997, 22, 919-921.
(7) Rosario, R.; Gust, D.; Hayes, M.; Jahnke, F.; Springer, J.; Garcia, A. A. Langmuir 2002, 18, 8062-8069.

(8) (a) Ikeda, T.; Nakano, M.; Yu, Y.; Tsutsumi, O.; Kanazawa, A. Adv. Mater. 2003, 15, 201-205. (b) Yu, Y.; Nakano, M.; Ikeda, T. Nature 2003, 425, 145. (c) Athanassiou, A.; Kalyva, M.; Lakiotaki, K.; Georgiou, S.; Fotakis, C. Adv. Mater. 2005, 17, 988-992.

(9) Berkovic, G.; Krongauz, V.; Weiss, V. Chem. Rev. 2000, 100, $1741-1753$

(10) Svelto, O. Principles of Lasers; Plenum Press: New York, 1989.

(11) Hide, F.; Diaz-García, M. A.; Schwartz, B. J.; Andersson, N. R.; Heeger, A. J. Science 1996, 273, 1833-1836.

(12) (a) Moses, D. Appl. Phys. Lett. 1992, 60, 3215-3216. (b) Tessler,

N.; Denton, G. J.; Friend, R. H. Nature 1996, 382, 695-697.

(13) (a) Tessler, N. Adv. Mater. 1999, 11, 364-370. (b) Kranzelbinder, G.; Leising, G. Rep. Prog. Phys. 2000, 63, 729-762.

(14) (a) Anni, M.; Gigli, G.; Paladini, V.; Cingolani, R.; Barbarella, G.; Favaretto, L.; Sotgiu, G.; Zambianchi, M. Appl. Phys. Lett. 2000, 77, 2458-2461. (b) Wu, C. C.; Li, Y. T.; Wong, K. T.; Chen, R. T.; Chien, Y. Y. Adv. Mater. 2004, 16, 61-65. (c) Chao, T. C.; Lin, Y. T.; Yang, C. Y.; Hung, T. S.; Chou, H. C.; Wu, C. C.; Wong, K. T. Adv. Mater. 2005, 17, 992-996.

(15) (a) Chibisov, A. K.; Görner, H. Chem. Phys. 1998, 237, 425-442. (b) Wojtyk, J. T.; Kazmaier, P. M.; Buncle, E. Chem. Mater. 2001, 13 , 2547-2551. (c) Görner, H. Chem. Phys. Lett. 1998, 282, 381-390. (d) Bohne, C.; Fan, M. G.; Li, Z. H.; Laing, Y. C.; Lusztyk, J.; Scaiano, J. C. J. Photochem. Photobiol., A 1992, 66, 79-90. (e) Malatesta, A.; Neri, C.; Wis, M. L.; Montanari, L.; Millini, R. J. Am. Chem. Soc. 1997, 119, 34513455 .

(16) Sheng, Y.; Leszczynski, J.; Garcia, A. A.; Rosario, R.; Gust, D.; Springer, J. J. Phys. Chem. B 2004, 108, 16233-16243.

(17) (a) Hobley, J.; Pfeifer-Fukumura, E.; Bletz, M.; Asahi, T.; Masuhara, H.; Fukumura, H. J. Phys. Chem. A 2002, 106, 2265-2270. (b) Rini, M.; Holm, A.-K.; Nibbering, E. T. J.; Fidder, H. J. Am. Chem. Soc. 2003, 125, $3028-3034$

(18) Görner, H. Chem. Phys. 1997, 222, 315-329.

(19) Levitus, M.; Talhavini, M.; Negri, R. M.; Atvars, T. D. Z.; Aramendia, P. F. J. Phys. Chem. B 1997, 101, 7680-7686.

(20) Abe, Y.; Nakao, R.; Horii, T.; Okada, S.; Irie, M. J. Photochem. Photobiol., A 1996, 95, 209-214.

(21) Shaklee, K. L.; Nahory, R. E.; Leheny, R. F. J. Lumin. 1973, 7, 284-309.

(22) Pisignano, D.; Mele, E.; Persano, L.; Gigli, G.; Visconti, P.; Cingolani, R.; Barbarella, G.; Favaretto, L. Phys. Rev. B 2004, 70, 205206. (b) Thompson, J.; Anni, M.; Lattante, S.; Pisignano, D.; Blyth, R. I. R.; Gigli, G.; Cingolani, R. Synth. Met. 2004, 143, 305-307.

(23) (a) McGehee, M. D.; Gupta, R.; Veenstra, S.; Miller, E. K.; DiazGarcia, M. A.; Heeger, A. J. Phys. Rev. B 1998, 58, 7035-7039. (b) Frolov, S. V.; Vardeny, Z. V.; Yoshino, K. Phys. Rev. B 1998, 57, 9141-9147. (c) Zenz, C.; Graupner, W.; Tasch, S.; Leising, G.; Iskra, K.; Flieser, J.; Neger, T. J. Appl. Phys. 1998, 84, 5445-5450. (d) Xia, R.; Heliotis, G.; Bradley, D. D. C. Appl. Phys. Lett. 2003, 82, 3599-3601. 